\title{
Programa de Salud Bucal en Escolares El Carmen, 1994 - 2007, Chile
}

\author{
Oral Health Program for El Carmen School Children 1994 - 2007, Chile
}

Ricardo Cartes-Velásquez* \& Alex Bustos Leal ${ }^{* *}$

\begin{abstract}
CARTES-VELÁSQUEZ, R. \& BUSTOS, L. A. Programa de salud bucal en escolares El Carmen, 1994 - 2007, Chile. Int. J. Odontostomat., 5(3):287-292, 2011.

RESUMEN: El Carmen era una comuna caracterizada por la pobreza, ruralidad y altos niveles de daño dental a principios de 1990. Esto llevó a que las autoridades locales y regionales junto a la Facultad de Odontología de la Universidad de Concepción desarrollaran un programa de salud bucal basado en el concepto de prevención temprana, ajustado a la realidad local y que promoviera la participación y empoderamiento de la comunidad en su salud. A lo largo de 13 años este programa ha sufrido varias modificaciones, principalmente de aumento y mejoramiento de coberturas, infraestructura y desempeño operacional. La cobertura e impacto partieron en valores cercanos al $10 \%$ durante el $1^{\circ}$ año hasta alcanzar el $100 \%$ en los últimos. Mientras que el COPD disminuyo de 7,38 en 1994 hasta llegar al 2,3 el 2007, muy cercano a las metas nacionales hacia el año 2010. Sin duda el programa ha mostrado ser exitoso en el mediano y largo plazo, esto gracias al compromiso de todos los actores involucrados, a la flexibilidad y permanente evolución mostrada para adaptarse a las condicionantes de la comunidad.
\end{abstract}

PALABRAS CLAVE: salud bucal, salud pública, rural, caries, Chile.

\section{INTRODUCCIÓN}

La caries y la enfermedad periodontal siguen mostrando una alta prevalencia a nivel mundial, con una polarización durante las últimas décadas, y donde los países no desarrollados han tenido un preocupante aumento tanto en la prevalencia como intensidad del daño bucal, contrastando con la disminución progresiva y sostenida mostrada en países desarrollados (WHO, 2002).

En el caso de Chile la prevalencia de caries en escolares alcanza a un $84,34 \%$ con un COPD de 3,43 (MINSAL, 2000a); situación que se ve agravada en la comuna de "El Carmen" con un 77\% y 7,38 respectivamente hacia el año 1994 (Hospital El Carmen, 2007).

Las políticas sanitarias nacionales han planteado como meta para el año 2010 disminuir el COPD a 1,9 (MINSAL, 2000b). Con el fin de lograr este objetivo se han desarrollado una serie de iniciativas tendientes a mejorar el actual estado de salud bucal de la población, entre estas destacan: Módulos Dentales de JUNAEB
(2004), Uso de Fluoruros (MINSAL, 1998), Atención dental garantizada a niños de 6 años dentro del Plan AUGE (MINSAL, 2005) y Escuelas Promotoras de Salud. Sin embargo, todas ellas han sido desarrolladas desde el nivel central y por lo tanto son sólo lineamientos generales que no siempre se ajustan a la realidad sociocultural propia de cada comunidad (WHO, 1986).

Desde esta perspectiva es que en la comuna de "El Carmen", a principios de los años 1990s y producto del desmejorado estado de salud bucal de su población, se comienza a gestar el desarrollo e implementación de un Modelo de Atención Dental, fuertemente asociado a la participación comunitaria (Bustos, 2000) y centrada en la Prevención dirigida hacia la población escolar.

El objetivo de este estudio es describir el desarrollo e implementación de este modelo además de evaluar la efectividad que ha tenido en el mejoramiento de la salud bucal de sus beneficiarios.

\footnotetext{
* Programa de Doctorado en Ciencias Médicas, Facultad de Medicina, Universidad de la Frontera, Temuco, Chile.

* Departamento de Prevención y Salud Publica Odontológica, Facultad de Odontología, Universidad de Concepción, Concepción, Chile.
} 
Características Sociodemográficas. La comuna de "El Carmen" se ubica en la provincia de Ñuble, octava región del Biobío, a $42 \mathrm{Km}$ de la ciudad de Chillán; y a $600 \mathrm{Km}$ de la capital Santiago de Chile. Según las proyecciones, la población comunal alcanzó a 15.458 habitantes en el año 2005, la cual se distribuye en $52,1 \%$ de hombres y $47,9 \%$ de mujeres, con una ruralidad de $75,3 \%$. La principal actividad económica es la agricultura y la comuna esta considerada como una de las 10 más pobres del país. En el año 2004 su IDH (Índice de Desarrollo Humano) fue de 0,611 y lo ubicó en el lugar 318 de 341 comunas, lo que significa un retroceso de 13 puestos con respecto a 1993 (MIDEPLAN - PNUD, 2005).

La atención de salud se otorga a través de un Hospital tipo IV, de baja complejidad, localizado en El Carmen, que cuenta con los servicios de medicina, pediatría y urgencia, con capacidad de 29 camas. Además, cuenta con un consultorio adosado que realiza las actividades de atención primaria a una población asignada de 7.359 personas. Existen además otros 12 establecimientos sanitarios de dependencia administrativa municipal: 5 postas rurales, 4 estaciones medico rurales, 1 unidad de manejo del riesgo cardiovascular, 1 centro kinésico y 1 clínica dental.

Desarrollo del Programa. Hacia el año 1990 El Carmen solo contaba con 1 odontólogo para satisfacer la demanda de atención de la comuna, la que presentaba un muy deteriorado estado de su salud bucal y una insuficiencia de recursos para poder revertir dicha situación. Las primeras medidas destinadas a mejorar esta situación consistieron en un aumento de las acciones preventivas desarrolladas en el hospital y la aplicación de flúor gel acidulado en las escuelas de manera periódica.

El año 1991 tras un diagnóstico participativo se determinó que una de las necesidades más sentidas por la comunidad era el de atención odontológica y era imperativo una intervención efectiva para solucionarlo. De esta manera se comenzó con el diseño del programa, con la asesoría del Departamento de Salud Publica de la Facultad de Odontología de la Universidad de Concepción, alineado con las políticas del Departamento Odontológico del Ministerio de Salud de Chile. Esta asesoría comenzó con la capacitación del personal de salud de El Carmen en materias de gestión y planificación, con el fin de desarrollar un plan estratégico para el mejoramiento de la salud bucal de la comunidad.
Así se comenzó a desarrollar la idea de una clínica dental destinada a la población escolar, con una modalidad de atención incremental y un fuerte componente promocional y preventivo. Este proyecto tuvo como pilar fundamental el apoyo transversal de todos los actores sociales de la comunidad.

De esta manera el municipio local realizó los aportes para la adquisición del instrumental y equipamiento necesario, además de la habilitación de la infraestructura de la futura clínica dental. La Universidad de Concepción donó 2 unidades dentales y comprometió la asistencia de alumnos internos para apoyar las actividades tanto asistenciales como promocionales. Mientras, el MINSAL destinó un odontólogo que se hiciera cargo de la implementación del programa. Por su parte, la comunidad se organizó para dar apoyo permanente a la gestión del programa.

En el año 1994 entra en funcionamiento la clínica dental con: 1 odontóloga, 1 asistente dental, secretaria, auxiliar de aseo y educadora de párvulos. En este año se ingreso al $77 \%$ de los escolares de $1^{\circ}$ básico, dando un $51 \%$ de altas; aumentándose dichas cifras año tras año.

El año 1996 con el fin de mejorar la cobertura de la población no beneficiada por la clínica dental, se logra la contratación de un $2^{\circ}$ odontólogo y 2 asistentes dentales para el Hospital, gracias a un aporte conjunto del Servicio de Salud Ñuble, I. Municipalidad de "El Carmen" y diversas organizaciones sociales.

En 1997 el aumento progresivo e inesperado de los pacientes tanto que ingresaban como los que se encontraban en control produjo un retraso de las metas proyectadas por lo que se hizo necesaria la contratación de un $3^{\circ}$ odontólogo y asistente dental para la clínica, financiado a través del proyecto gubernamental Fondo Nacional para la Superación de la Pobreza.

Lo anterior se vio reforzado al año siguiente con la contratación de 1 odontólogo más y la implementación de métodos de atención innovada, trabajo a 6 manos. En esta fecha también se comenzó con la implementación de los "Cepilleros", salas especiales para la promoción y educación en salud bucal, gracias al financiamiento de diversos proyectos y el apoyo de padres y apoderados de los respectivos colegios. 
Con respecto a la participación comunitaria esta se ha visto respaldada por la creación de la organización "Amigos de la Clínica Dental", formada por padres, apoderados y cercanos al quehacer odontológico, que apoyan principalmente la labor educativa y promocional de la clínica.

Durante el año 2001 se produjo la adquisición de un bus escolar que facilita el transporte de los niños de sectores rurales y el cambio de sede de la Clínica Dental a nuevas dependencias pasando a ser el "Modulo Dental El Carmen", el que el 2003 ha logrado la implementación de su sala educativa lo que permite la realización de actividades educativas de manera formal y programada.

Modelo de Atención. Este se basa en la recuperación, mantención y educación en las conductas de autocuidado de la salud bucal, a través de una serie de actividades restauradoras, preventivas y promocionales de los escolares de $1^{\circ}$ a $8^{\circ}$ básico de la comuna de "El Carmen".

Así es como todos los niños que comienzan la enseñanza básica son ingresados al Programa de Atención Dental donde se realiza:

1. Actividades Restauradoras

- Obturaciones.

- Endodoncias.

- Extracciones.

2. Actividades Preventivas.

- Profilaxis.

- Sellantes.

- Fluoraciones.

3. Actividades Promocionales.

- Educación de Higiene General.

- Técnica de Cepillado.

Educación a los padres y apoderados. Una vez que el escolar ha recuperado un buen estado de salud bucal y ha adquirido las condiciones biológicas y conductuales para preservar dicho estado se le da el "Alta Integral". A medida que este continúa su periodo de enseñanza básica es mantenido en controles anuales, donde se realizan las acciones necesarias para preservar su condición de salud bucal, pasando a obtener un "Alta de Mantención" finalizado este control.

Cabe destacar además que, el Programa se ve reforzado en las escuelas con acciones periódicas, tales como: Enjuagatorios, talleres educativos y el trabajo en los "Cepilleros"; coordinadas por los dentistas del Módulo Dental y realizadas por los profesores de cada establecimiento, previamente capacitados en estas materias.

\section{RESULTADOS}

La evaluación se realizó mediante 3 indicadores:

1.- Cobertura: Es el porcentaje de pacientes ingresados a tratamiento en relación a la población objetivo del programa.

2.- Impacto: Es el porcentaje de pacientes dados de alta en relación a la población objetivo del programa.

3.- COPD: Número de piezas con historia de daño por caries.

Como se observa en la Tabla I se han aumentado los porcentajes de cobertura e impacto en forma sostenida a lo largo de los años. Debido al éxito del programa y al lograr las metas sanitarias con el grupo etario planificado se pudo extender esta atención a otros grupos etarios no contemplados originalmente, alcanzando a cubrir a la totalidad de la población escolar de la comuna.

En la Figura 1 podemos apreciar los resultados concretos del programa en la disminución del daño bucal de sus beneficiarios. Finalmente la Figura 2 muestra el cambio de rumbo que ha tenido el Programa, pasando desde un comienzo fuertemente restaurador a un estado actual eminentemente promocional y preventivo, que es el objetivo principal de este modelo.

Tabla I. Porcentaje de Cobertura e Impacto del Programa de Atención Dental en escolares de enseñanza básica de la Comuna de "El Carmen”, Chile. 1994 - 2007.

\begin{tabular}{lccccccc}
\hline \multirow{2}{*}{ Indicadores } & $\mathbf{7}$ Años \\
\cline { 2 - 8 } & $\mathbf{1 9 9 4}$ & $\mathbf{1 9 9 7}$ & $\mathbf{1 9 9 9}$ & $\mathbf{2 0 0 1}$ & $\mathbf{2 0 0 3}$ & $\mathbf{2 0 0 5}$ & $\mathbf{2 0 0 7}$ \\
\hline Cobertura & 8 & 17,2 & 82,3 & 93 & 99 & 119,4 & 121,6 \\
Impacto & 4 & 12,1 & 19,5 & 59,6 & 100 & 100 & 100 \\
\hline
\end{tabular}




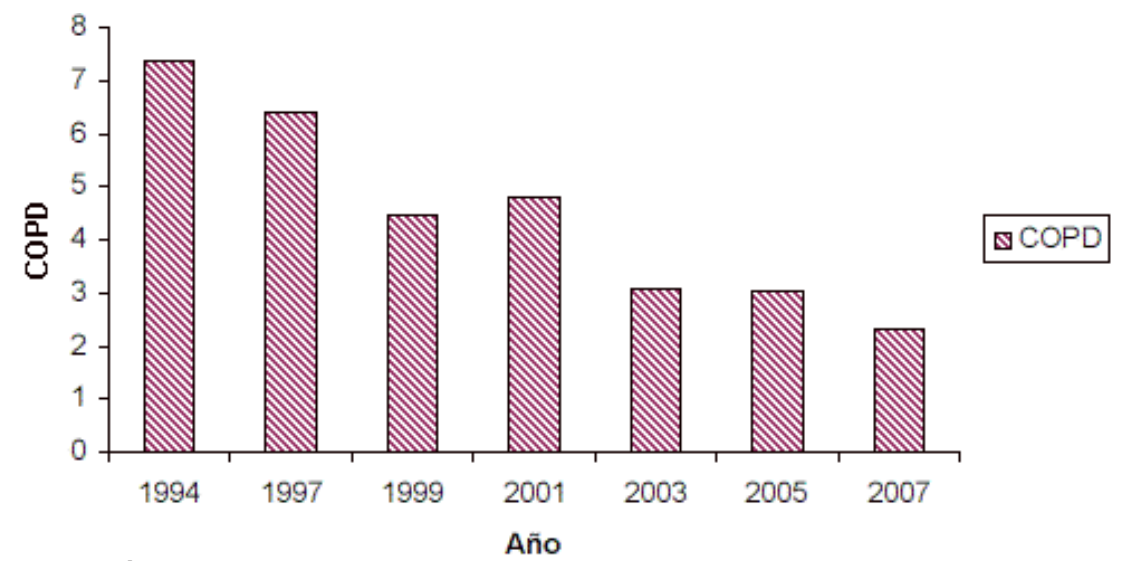

Fig. 1. Índice COPD en niños y niñas de 12 beneficiarios del Programa de Atención Dental de la Comuna de "El Carmen", Chile. 1994 - 2007. Chile.

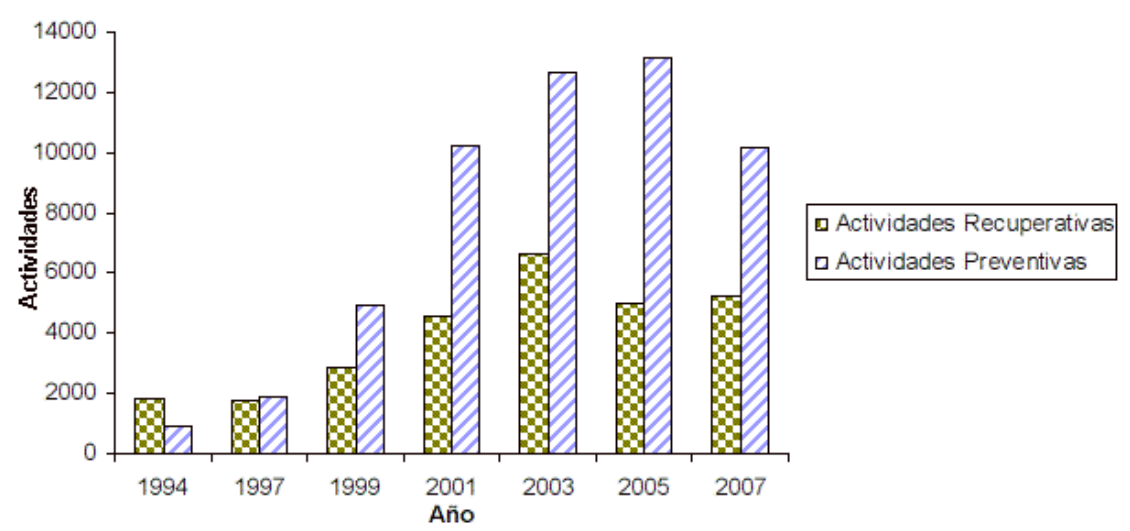

Fig. 2. Relación de Actividades Recuperativas y Preventivas del Programa de Atención Dental de la Comuna de "El Carmen", Chile. 1994 - 2007.

\section{DISCUSIÓN}

Como en muchos países la implementación de diversos Oral Health Programmes (OHPs) buscan un mejoramiento del estado de salud bucal de su población entendiendo las repercusiones que tiene esta en la calidad de vida de las personas (AI Shamrany, 2006). Sin embargo diferencias en las condiciones socioeconómicas, estilos de vida, aspectos locales y concepciones de ser, hacer o mantener salud originan una amplia gama de programas no existiendo un modelo que sea perfecto para todas las condiciones posibles.

Aún así existe una línea o aspecto transversal a la mayoría de los programas, desde las experiencias danesas (Poulsen \& Risager, 1975; Downer et al., 1983; Bille, 1980) de finales de los 60 hasta las que se desarrollan en Asia (Hartono et al., 2002; Peng et al., 2004) y África (Frencken et al., 2001; van Palestein Helderman et al., 1997) en la última década, y que se refiere al concepto de "prevención temprana", este pareciera ser una idea acotada a márgenes bien definidos, sin embargo, no representa más que una guía que es interpretada y llevada a la práctica de diversas formas.

Este concepto, como lo indica su nombre, presenta dos dimensiones a considerar; la primera de ellas de que manera se implementara la "prevención" y la segunda que tan "temprana" será esta. De esta manera nos encontramos con un abanico de OHPs que varían desde modelos exclusivamente educativos hasta modelos asistenciales-curativos subsidiados (Tubert-Jeannin et al., 1998) y un espectro que muestra en distinta proporción la mixtura de la Oral Health Education y el Oral Health Care transitando desde una prevención primaria a una terciaria; y que en la variable etaria va desde infantes a adolescentes.

Ante tal variedad de modelos basados en este concepto base de "prevención temprana" y considerando además el análisis de "costo efectividad" que requiere todo programa en salud pública, la pregunta que cabe hacerse es: ¿Cuál modelo es el que rinde más? Y ante ella la evidencia actual muestra que de todos los tipos de experiencias, son aquellas que unen tanto el aspecto preventivo promocional como el curativo asistencial de manera estructurada, coherente y permanentemente monitoreadas las que obtienen los mejores resultados a largo plazo; y aquellas experiencias que se centran solo en educación con un periodo de intervención corto solo logran un cambio pasajero en las conductas de autocuidado que no se reflejan en un mejoramiento concreto de los indicadores en salud bucal (Kay \& Locker, 1996, 1998).

Ante este panorama parece obvio, al menos, en pos de los resultados, que las políticas en salud bucal debiesen ser más agresivas 
y asistencialistas hasta provocar un cambio en las conductas de autocuidado de las actuales y futuras generaciones, convirtiéndolas desde acciones planificadas y obligatorias en hábitos propios de la vida cotidiana, sobre todo en los países en vías de desarrollo. Pero este cambio requiere que la población comprenda, valore, integre y alcance un nivel de compromiso y participación con los objetivos y acciones que desarrollan estos modelos, con el fin de que se conviertan en actores principales de su proceso de salud y no en meros receptores de ella. Este hecho se hace evidente en el modelo de "El Carmen" donde la intervención prolongada ha logrado crear un background social que sostiene las conductas de autocuidado en salud bucal de sus beneficiarios y legitima la existencia y funcionamiento continuo del Módulo Dental, respaldada por la creación de la organización "Amigos de la Clínica Dental".

Otro importante factor a considerar es la presencia de un ente técnico y de carácter privado que apoye y asesore metodológicamente el modelo desde su creación hasta su implementación; que en este caso ha sido la Facultad de Odontología de la Universidad de Concepción. Esto ha permitido el desarrollo de un modelo personalizado, acorde a las características de la comunidad y que se sustenta en bases científicas y la evidencia actual que existe con respecto a estos modelos de salud. Además de una progresiva adaptación a los nue- vos desafíos y condicionantes; donde el odontólogo destaca como un líder para promover cambios desde la propia comunidad.

En conclusión, como lo ha señalado la OMS (Frazier et al., 1983; Petersen \& Kwan, 2004; Petersen, 2005) en sus últimos surveys y workshop internacionales la evidencia respecto a cuales representan los programas de salud bucal que cumplan a cabalidad con los criterios de efectividad y eficiencia se encuentran aún no establecidos, por esto se hace necesario la verificación de las implementaciones y resultados que presentan los distintos programas desarrollados a nivel global, regional y local.

En el caso de El Carmen, el programa ha demostrado su efectividad en el mejoramiento de la salud bucal de sus beneficiarios, logrando además objetivos como el empoderamiento de la comunidad, conservando su enfoque en la promoción en salud. Sin duda, falta la evaluación de la relación costo/efectividad para ofrecer una visión más completa del programa.

El éxito de este programa se basa en un adecuado cumplimiento de todas sus etapas, con asesoría técnica, apoyo institucional, participación y compromiso local junto a una permanente adaptación al entorno y condicionantes que exige el medio local.

CARTES-VELÁSQUEZ, R. \& BUSTOS, L. A. Oral health program for El Carmen school children 1994 - 2007, Chile. Int. J. Odontostomat., 5(3):287-292, 2011.

ABSTRACT: In 1990 the rural community of "El Carmen" presented high levels of poverty and dental damage. This led local and regional authorities to join forces with the Dental Faculty of Universidad de Concepción to implement an oral health program based on the concept of early prevention. This program was designed to fit the local reality, promoting participation of the community and strengthening their health. For 13 years the program has undergone several modifications, which have mainly increased and improved coverage, infrastructure, and operational performance. The initial coverage and impact was near $10 \%$ and in recent years, has reached $100 \%$ of the community. DMFT index has dropped gradually and significantly; in 1994 it was 7.38 and in 2007 it was 2.3. The latter value is very close to national goal set for 2010. Clearly, this program has proven to be succesfull in the mid- and long-term. This is due to the commitment shown by all the professionals involved in the program as well as its flexible nature and constant improvement, which has allowed it to adapt to local reality.

KEY WORDS: oral health, public health, rural, caries, Chile.

\section{REFERENCIAS BIBLIOGRÁFICAS}

Al Shamrany, M. Oral health-related quality of life: a broader perspective. East. Mediterr. Health J., 12:894-901, 2006.

Bille, J. Dental caries in a group of 20 -year-olds after previous participation in public child dental health services in Copenhagen, Denmark. Community Dent. Oral Epidemiol., 8:27-32, 1980.
Bustos, A. Conceptos, Fundamentos y Desafíos para la Atención Primaria en Salud. 1a Ed. Concepción, Universidad de Concepción, 2000.

Downer, M. C.; Davies, G. N. \& Holloway, P. J. The Danish oral health care service for children: an evaluation. Int. Dent. J., 33:231-7, 1983. 
Frazier, P. J.; Johnson, B. G. \& Jenny, J. Health educational aspects of preventive dental programs for school-age children in 34 countries--final results of an FDI international survey. Int. Dent. J., 33:152-70, 1983.

Frencken, J. E.; Borsum-Andersson, K.; Makoni, F.; Moyana, F.; Mwashaenyi, S. \& Mulder, J. Effectiveness of an oral health education programme in primary schools in Zimbabwe after 3.5 years. Community Dent. Oral Epidemiol., 29:253-9, 2001.

Hartono, S. W.; Lambri, S. E. \& van Palenstein Helderman, W. H. Effectiveness of primary school-based oral health education in West Java, Indonesia. Int. Dent. J., 52:13743, 2002.

Hospital El Carmen. Registro atención odontológica 1993 2006. El Carmen, Ilustre Municipalidad El Carmen, 2007.

Junta Nacional de Auxilio Escolar y Becas (JUNAEB). Análisis con enfoque de género de la población escolar evaluada para la asignación del programa de alimentación escolar y programa de salud del estudiante de JUNAEB. 1a Ed. Santiago, JUNAEB, 2004.

Kay, E. J. \& Locker, D. Is dental health education effective? A systematic review of current evidence. Community Dent. Oral Epidemiol., 24:231-5, 1996.

Kay, E. \& Locker, D. A systematic review of the effectiveness of health promotion aimed at improving oral health. Community Dent. Health, 15:132-44, 1998.

MIDEPLAN - PNUD. Las trayectorias del Desarrollo Humano en las Comunas de Chile (1994-2003). Temas de Desarrollo Humano Sustentable número 11. Santiago, MIDEPLAN, 2005.

MINSAL. Normas de uso de Fluoruros en Odontología. $1^{\text {a }}$ Ed. Santiago, MINSAL, 1998.

MINSAL. Perfil Epidemiológico en Salud Bucal. $1^{\text {a }}$ Ed. Santiago, MINSAL, 2000a.

MINSAL. Lineamientos Estratégicos Plan de Salud Buco dental 2000 - 2010. $1^{\text {a }}$ Ed. Santiago, MINSAL, 2000b.

MINSAL. Garantías Explicitas en Salud. Guía Clínica Salud Oral Integral en Niños de 6 años. $1^{\text {a }}$ Ed. Santiago, MINSAL, 2005.

Peng, B.; Petersen, P. E.; Bian, Z.; Tai, B. \& Jiang, H. Can school-based oral health education and a sugar-free chewing gum program improve oral health? Results from a two-year study in PR China. Acta Odontol. Scand., 62:328-32, 2004.

Petersen, P. E. \& Kwan, S. Evaluation of community-based oral health promotion and oral disease prevention--WHO recommendations for improved evidence in public health practice. Community Dent. Health, 21:319-29, 2004.

Petersen, P. E. Priorities for research for oral health in the 21st century--the approach of the WHO Global Oral Health Programme. Community Dent. Health, 22(2):714, 2005.

Poulsen, S. \& Risager, J. Effect on dental caries of a dental public health program for Danish schoolchildren. Community Dent. Oral Epidemiol., 3:161-5, 1975.

Tubert-Jeannin, S.; Morel-Papernot, A. \& Woda, A. Evaluation of a dental benefit plan for children conducted in Auvergne, France, since 1992. Community Dent. Oral Epidemiol., 26:272-82, 1998.

van Palenstein Helderman, W. H.; Munck, L.; Mushendwa, S.; van't Hof, M. A. \& Mrema, F. G. Effect evaluation of an oral health education programme in primary schools in Tanzania. Community Dent. Oral Epidemiol., 25:296300, 1997.

Worl Health Organization (WHO). Ottawa Charter for Health Promotion. First International Conference on Health Promotion. Ottawa, World Health Organization, 1986.

World Health Organization (WHO). Global Oral Health Data Bank. Geneva, World Health Organization, 2002.

Dirección para correspondencia:

Ricardo Cartes-Velásquez

Los Olivos 37, Penco

CHILE

Email: ricardo@cartesvelasquez.com

Recibido : 09-10-2011

Aceptado: 10-10-2011 